\title{
SOP 14: Population Pharmacokinetic Analysis
}

\section{Background and Objectives}

Pharmacokinetic studies are an essential component of drug development (see 'SOP 13: Pharmacokinetic Data Analysis'). The relevant data are usually obtained from groups of animals, healthy volunteers, or patients. 'Population Pharmacokinetics' refers to a method of pharmacokinetic data analysis that is especially suited to data from groups of individuals, or 'populations'. The objective of population pharmacokinetic analyses is to estimate not only the typical (mean) pharmacokinetic parameters of a population but also to obtain quantitative (and statistically correct) information on the extent and the sources of pharmacokinetic variability. This is useful for assessing the probability of drug concentrations that will be below or above the therapeutic range with a uniform dosing scheme, for setting up dose recommendations for subpopulations with altered pharmacokinetics, and as a basis for adaptive individual dosing in the case of drugs with a narrow therapeutic range.

The extent of pharmacokinetic variability between and within individuals is often not obvious to non-specialists. Individual clearances as determined in groups of 20-30 individuals usually vary by a factor of $4-5$ or more; the same variability, then, exists in the total area under the plasma concentration-time curve $(A U C)$ and in the concentration at steady state $\left(C_{s s}\right)$ under continuous dosing. Both $A U C$ and $C_{s s}$ are related to tumor response and toxicity [1-4]. Thus, the analysis of pharmacokinetic variability can help understand why therapy fails in individual cases and is a prerequisite for an individual optimization of drug therapy.

The basic paramaters of a population model are population mean and interindividual and residual variance parameters. These are fitted simultaneously. A population analysis does not require an analysis of individual kinetic profiles (even though it allows for this). Even sparse data, like only one or two concentration measurements per patient, can be analyzed. Variations of dose, route of administration, and dosing and sampling schedule within the same study are not a problem. Thus, this method is particularly useful with data which are obtained under incompletely controlled conditions in clinical routine care.

The successful application of population models requires a solid grasp of pharmacokinetic and statistical concepts and considerable technical experience with specialized software. Experience shows that, for a beginner, it will usually take several months of full-time work to become productive in this field.

This document concentrates on standard procedures for data analysis. The US Food and Drug Administration has issued a guidance on population pharmacokinetics [5] containing a broader discussion of scientific and regulatory issues and additional recommendations, especially on study design and execution and on data handling.

\section{Study Design and Execution}

Standard procedures have been described in 'SOP 13: Pharmacokinetic Data Analysis', section 'Study Considerations' and in [5, 6]. A few points are emphasized here.

The design of a study that will be followed by a population analysis may be less rigorous than that of a study where the data will be analyzed for each individual separately. Dosing and sampling schedules may vary between individuals. There is no need for discrete dose groups; doses may be varied on a continuous scale in order to check whether pharmacokinetics are linear over the entire dose range. No separate groups of patients with pronounced hepatic or renal function impairment need to be recruited in order to look for a dependence of pharmacokinetics on organ function. Patients with a wide range of hepatic or renal function parameter values must be included for this purpose but the variation may be continuous as typically found in non-selected patients of a phase III or phase IV study.

Even though a population analysis can accommodate sparse data, some extensive sampling data are needed for

\section{KARGER}

Fax +497614520714

E-mail Information@Karger.de

www.karger.com
(C) 2003 S. Karger GmbH, Freiburg 
determining the appropriate 'structural' model (how many compartments, first-order versus zero-order absorption, lag time yes/no etc.). These data may come from a previous study. If a study with sparse sampling is planned, then the question whether pharmacokinetics will be linear over the entire range of doses to be applied should have been investigated and answered in a preceding study with complete concentration-time profiles, e.g. a phase I study. If such data are available it is useful to include them in the population analysis of sparse data from a subsequent study.

The sampling times should cover three or more half-lives during the terminal phase of disposition. Optimal sampling time points should be determined if results from a study with extensive sampling are available. The 'optimal' time points, in this context, are those where the measurements are most informative with regard to the pharmacokinetic parameters of interest (most often clearance) [7, 8]. These time points should be translated into 'time windows' if the data are to be acquired in a clinical setting (e.g. 4-6 h, 22-28 h); this will prevent the temptation of recording false sampling times when samples could not be drawn at the exact scheduled time. The effect of inaccurately documented sampling or dosing times, typically an inflation of residual variation, is even more detrimental in a population analysis than in a conventional analysis because false data from only one or few individuals will affect the parameter estimation for the entire population. A documentation of infusion times and rates which is just copied from the study design can be grossly inaccurate like, for example, in the case of major fluctuations in the infusion rate, or, when an infusion by intravenous drip came to completion well ahead of the scheduled time. Infusion pumps should be employed whenever possible to eliminate this source of errors.

Studying patients on more than one occasion, e.g. during more than one cycle of chemotherapy, may allow one to distinguish the variability between different occasions within the same patient from the variability between patients. Pharmacokinetic variability will all appear as being interindividual when only data from single occasions are available [9]. This may result in overly optimistic expectations as to the benefit from dose adjustments that are based on individual plasma concentration measurements [5, 9].

\section{Data Acquisition and Handling}

See 'SOP 13: Pharmacokinetic Data Analysis', section 'Data Handling', and [5] for appropriate procedures. A few selected points are emphasized here.

Information on covariates, in addition to sex, race, age, weight and height, such as concomitant diseases, co-medication, smoking habits, should be collected rather exten- sively as it may become an explanatory variable later in the modeling process. Weights and heights should be measured rather than found out by asking the patient. Weight measurements should be repeated in cancer patients who are monitored for more than one cycle of chemotherapy. ASCII tables with blanks or commas as field separators are most easily transferable between databases and modeling programs. All data, including the covariate information, should be sorted by individual and by time rather than being stored in separate blocks.

The exclusion of faulty data is even more important in population analyses than in individual modeling because grossly false data in one individual (like plasma concentration values that keep rising after the documented end of an infusion) can jeopardize the success of the analysis for the entire population. As pointed out in SOP 13, exclusion criteria for faulty data should be defined before modeling starts. Excluding these data before modeling starts will also save a great deal of time by avoiding the repeated fitting of identical models to slightly differing data sets. It will also prevent the temptation to modify the exclusion criteria in order to make the data fit an inappropriate model. (An 'inappropriate model' is also one that underestimates residual variability because exclusions have been made in hindsight to 'improve' the fit.) All exclusions must be documented.

Assembling concentration data while a study is still running and doing a preliminary model analysis of the data, say, of the first 20-30 patients will help in detecting sources of erroneous data or information gaps at an early stage.

\section{Data Analysis}

\section{Preparatory Graphical Analysis}

Extensive graphical analyses should precede every population model analysis. This is the basis for devising the 'structural model'; the structural model is the 'kinetic' part of the population model, like, for instance, a two-compartment linear model, or a two-compartment model with saturable elimination. It is later assumed to hold for all individuals even though the individual data will often not contain enough information to support this assumption.

All concentration-time data should be plotted by individual (one plot for each individual) to check for implausible data and to gather information on the number of disposition phases in individuals with extensive sampling. These plots may also indicate that a lag time parameter will be needed. Linearity should be checked for by a plot of individual $A U C$ s versus dose if a subset of individuals with complete concentration-time profiles is available (use the trapezoidal rule to calculate $A U C$ s, see 'SOP 13: Pharmacokinetic Data Analysis', section 'Noncompartmental 
Data Analysis'). If such data are not available, the decision whether to use a linear or a non-linear structural model will have to be based on data from a preceding study with extensive sampling (e.g. phase I), or on information from the literature. Both linearity and the number of disposition phases should be examined in a semi-logarithmic plot of all individual concentration-time data for treatment groups with a similar mode of administration (dosing at about the same time point by the same route; same duration of infusions); each individual should be represented by one line ('spaghetti plot'). A trend in the terminal elimination phase to be flatter in individuals with high concentrations may indicate saturable elimination even when only one dose or a very narrow range of doses was used in the population. Median concentration-time profiles should be plotted for separate dose groups where applicable. A robust smoother, like LOWESS [10], may be substituted for the median concentrations when the number of individuals varies between sampling time points. A plot of median plasma concentrations versus time by dose group, where concentrations are expressed as fractions of dose per unit volume ('dose-normalized'), should be made when a similar mode of administration was applied over a range of doses in order to check whether the location of these curves is independent of dose.

\section{Basic Model}

A population model is a combination of a 'structural model' (see preceding section) with models of interindividual and residual variability. An important goal of pharmacokinetic population analyses (often the most important one) is the identification of predictable (non-random) components of interindividual variability (depending on covariates like sex, age, co-medication etc.). This goal is achieved in two steps: The data are first described by a model where all variability between individuals is assumed to be random ('basic' or 'descriptive' model). In a second step, the results are graphically examined for systematic effects of covariates and a sequence of refined models of interindividual variability is tested which includes effects of covariates.

\section{Structural Model}

The structural model is the deterministic part of a population model. It defines the pharmacokinetic processes behind the data much in the same way as do the traditional models for individual concentration-time profiles (cf. 'SOP 13: Pharmacokinetic Data Analysis', sections 'Compartmental Data Analysis', 'PBPK Models'; and 'PK/PD Modeling'). The number of disposition phases assumed for the population should usually be the maximum number that was found in any individuals in the preparatory graphical analysis (section 'Data Analysis'). The structural model must be non-linear if the 'spaghetti plot' or the plot of $A U C$ by dose group indicates non-linearity even if there is no individual concentration time profile that exhibits non-linearity. The population approach will allow to estimate mean population parameters of a non-linear model (like mean $v_{\max }$ or mean $k_{M}$ ) in this case while the more traditional approach of fitting individual profiles one by one would have a problem of overparameterization.

Structural pharmacokinetic models should be expressed in terms of 'primary pharmacokinetic parameters' [11], i.e. clearances and volumes, rather than rate constants because is is then easier to set up hypotheses about the influence of covariates like weight, sex, age, or of concomitant diseases which, for example, alter hepatic or renal function or plasma protein concentration.

\section{Interindividual Variation}

As said before, the first step in a population analysis is to treat all interindividual variability as random. Individual kinetic parameters are only modeled as functions of population means and individual random deviations at this stage. The most commonly used basic model for individual clearance, for example, is the following:

$$
C L_{i}=\overline{C L} \cdot e^{\eta_{C L, i}}
$$

where $C L_{i}$ is the clearance of individual $i, i=1, \ldots, n$ ( $n=$ total number of individuals), $\overline{C L}$ is the mean population clearance, and $\eta_{C L}$ is a random variable with mean zero and variance $\omega_{C L}{ }^{2}$. This model implies that clearance cannot be negative and, thus, is preferable over models like $C L_{i}=\overline{C L}+\eta_{C L, i}$. Also, if the distribution of $\eta_{C L}$ is symmetrical, the exponential model implies that the distribution of individual clearances is skewed to the right which is a commonly observed feature of distributions of pharmacokinetic parameters [12]. The models for distribution volumes and distribution clearances, as well as the models for lag time and first- or zero-order constants of absorption, should bet set up similarly.

Weight is the only covariate that may be incorporated in the basic model of interindividual variability. Both theoretical reasons [13] and a large body of empirical evidence [14] justify the assumption that, in principle, volumes are proportional to weight and clearances are proportional to the 3/4 power of weight. Making this assumption part of the basic model will facilitate the identification of influential covariates other than weight in the second step. For example, systematic deviations of individual clearances from the expected dependence on weight may then allow one to identify an influence of sex, age, or plasma protein concentration which might go unnoticed if the dependence on weight is assigned unlimited flexibility so that it is able to describe too much of interindividual variability. The decision whether to incorporate weight as a covariate in the basic model should be made before the data analysis is started. Individual weights should always be related to a standard weight, like $70 \mathrm{~kg}$. This leads to the following
Standard Operating Procedures for Clinical Trials of the CESAR Central European Society for Anticancer Drug Research - EWIV 
form of the models for the interindividual variability of clearances and volumes if weight is part of the basic model:

$$
\begin{gathered}
C L_{i}=\overline{C L} \cdot\left(\frac{\mathrm{BW}_{i}}{70 \mathrm{~kg}}\right)^{0.75} \cdot e^{\eta_{C L, i}}, \\
V_{i}=\bar{V} \cdot \frac{\mathrm{BW}_{i}}{70 \mathrm{~kg}} \cdot e^{\eta_{V, i}},
\end{gathered}
$$

where $\mathrm{BW}_{i}$ is the individual body weight expressed in $\mathrm{kg}$. In the example, the estimates of $\overline{C L}$ and $\bar{V}$ represent the mean clearance and distribution volume of 'typical' subjects weighing $70 \mathrm{~kg}$.

Body weight, as determined by weighing on a scale, may be replaced by 'lean body weight', a figure calculated from height and weight for a better representation of the total mass of the tissues which determine drug disposition:

$$
\begin{aligned}
& \operatorname{LBW}_{\text {men }}=1.10 \cdot \text { weight }(\mathrm{kg})-128 \cdot(\text { weight }(\mathrm{kg}) / \operatorname{height}(\mathrm{cm}))^{2}, \\
& \mathrm{LBW}_{\text {women }}=1.07 \cdot \text { weight }(\mathrm{kg})-148 \cdot(\text { weight }(\mathrm{kg}) / \operatorname{height}(\mathrm{cm}))^{2} .
\end{aligned}
$$

Body surface area (BSA), on the average, is proportional to $\mathrm{BW}^{2 / 3}=\mathrm{BW}^{0.67}$. Thus, applying a fixed dose per unit BSA is roughly the same as dosing in proportion to clearance under the model of Eq. 2.

The above model for $C L_{i}$ and $V_{i}$ and similar models for other individual kinetic parameters, are composed of 'fixed effects', like $\overline{C L}, \bar{V}$ and $\mathrm{BW}_{i}$, and of 'random effects', like $\eta_{C L, i}$ and $\eta_{V, i}$. This is why population models, as defined here, are also often called 'mixed-effects' models.

Individual clearance and volume parameters may be correlated for reasons other than body weight. For example, for a low-extraction drug that is strongly bound to plasma albumin, both clearance and distribution volume will be roughly proportional to the fraction unbound in plasma and, hence, will be correlated. The basic model of interindividual variability should then include an additional floating parameter for the covariance of the ' $\eta$ 's' on clearance and volume, $\omega_{C L, V}$.

The number of possible variance and covariance parameters quickly increases with the number of structural parameters in the population model. For example, a one-compartment model with first-order absorption and lag time, having four structural parameters, could have up to ten interindividual variance and covariance parameters. Given the extent of residual variation that is usually encountered in pharmacokinetics, no more than about one half of all possible interindividual variance and covariance parameters will be identifiable. The decision which ones to select for the basic population model is somewhat arbitrary. Clearance and the central volume of distribution should always be assigned an interindividual variance parameter. Covariance parameters should only be used where a covariance has to be expected a priori. It will hardly be possible to estimate an interindividual variance for both a rate constant of absorption and lag time. An initial fit of the basic interindividual variance model will often give an estimate close to zero for some of the interindividual variance or covariance parameters. These should then be fixed to zero to make the model more stable.

A useful model for the interindividual variability of bioavailability, $F$, is the following:

$$
F_{i}=\frac{1}{1+e^{-\ln (\bar{F} /(1-\bar{F}))+\eta_{F, i}}},
$$

where $F_{i}$ is the bioavailability in individual $i$ and $\bar{F}$ is the mean bioavailability, i.e. the value of $F$ that results if $\eta_{F, i}$ is zero. The equation represents a 'logistic' model [15]; it implies that $F_{i}$ varies from 0 to 1 as $\eta_{F, i}$ varies from $-\infty$ to $+\infty$.

The total number of interindividual variance and covariance parameters should be reduced as long as the objective function (usually a log-likelihood) does not deteriorate significantly. Changes in the log-likelihood should be tested for significance using the likelihood-ratio test [16] with $\alpha \leq 0.05$.

\section{Residual Variation}

A 'multiplicative' model should be used for residual variability if the measured observations span more than one order of magnitude:

$$
y_{i j}=\widehat{y_{i j}} \cdot\left(1+\varepsilon_{i j}\right),
$$

where $y_{i j}$ is the observed concentration in individual $i$ at time $j, \widehat{y_{i j}}$ is the 'mixed-effects predictions' as derived from the individual parameters $\left(V_{i}, C L_{i}\right.$ etc.) and $\varepsilon$ is another random variable with mean zero and variance $\sigma^{2}$. The variance $\sigma^{2}$ will practically always have to be assumed to be the same in all patients. The above residual variance model implies a constant coefficient of variation over the entire observable concentration range. With this model, care must be taken that the data set has no observation where the predicted concentration is zero, e.g. at the time of the first dose, because the predicted residual variance for this observation will be zero, causing a division by zero in computing the likelihood. Note that, with the above model, the expected residual, $\widehat{y_{i j}} \cdot \varepsilon$, is proportional to the predicted concentration. Weighting the observations such that the weights depend on the observations themselves, e.g. $w_{i j}=1 / y_{i j}$ or $w_{i j}=1 / y_{i j}^{2}$, is discouraged because it causes the weights to be random variables which has been shown to result in imprecise parameter estimates [17].

Semi-logarithmic plots of measured concentrations versus time will sometimes exhibit a residual variation that 'shrinks' at higher concentrations; a model where the residual error is proportional to the square root of the predicted concentration is then preferable:

$$
y_{i j}=\widehat{y_{i j}}+\sqrt{\widehat{y_{i j}}} \cdot \varepsilon_{i j} \text {. }
$$


This model implies that the variance rather than the standard deviation of the observations is proportional to the model prediction.

An additive residual variance model:

$$
y_{i j}=\widehat{y_{i j}}+\varepsilon_{i j}
$$

should only be considered when the measurements to be modeled all fall within one order of magnitude.

\section{Explanatory Model}

An important objective of pharmacokinetic population analyses is to discover relationships between observable covariates and individual kinetic parameters which explain part of the interindividual variability that has all been ascribed to random effects in the basic model. One such relationship may already have been incorporated in the basic model: the body weight dependence of clearance and volume parameters. If it has not been part of the basic model it should be tested as the first covariate in building the explanatory model. Other covariates which often influence individual kinetic parameters in a systematic, predictable way are: age, sex, plasma protein concentration, impairment of hepatic or renal function by concomitant diseases, co-medication, smoking habit, race, genetically determined deficiency of drug-metabolizing enzymes. Quantitative modeling of the influence of cavariates is of special interest in drug development because it will allow one to determine whether subgroups of patients need special dosage recommendations. For drugs with a narrow therapeutic range which require continuous monitoring of individual plasma concentrations or effects, models of covariate relationships help determine the best starting dose for the individual patient.

Hypotheses about the influence of covariates should be derived from general pre-existing pharmacokinetic knowledge and from the results of earlier studies of the compound under investigation. For example, a possible dependence of drug clearance on individually determined creatinine clearance should be tested if a compound is known to be excreted unchanged to an appreciable extent. Or, the influence of a co-medication should be investigated if it is known to induce or inhibit one of the primary metabolizing enzymes. Another example is the influence of plasma albumin on the distribution volume of a drug which is highly albumin-bound (also on clearance if it is a low-extraction drug). Hypotheses testing should start with estimating the individual deviations from the mean population parameters (' $\eta$ 's', see Eqs. 2, 3) by a Bayesian procedure (for example, the POSTHOC function of NONMEM [18]). The individual deviations should be plotted versus the hypothesized influential covariates in order to check whether there is any trend in accordance with the hypoth- esis. If so, a fixed dependence of the pharmacokinetic parameter in question on the individual covariate values should be incorporated in the population model by introducing a 'shift parameter'. As an example, a dependence of drug clearance on individual creatinine clearance may be modeled in the following way:

$$
\begin{aligned}
C L_{i}= & \overline{C L} \cdot\left(\mathrm{BW}_{i} / 70\right)^{0.75} \\
& \times\left(1-\theta \cdot\left(120 \mathrm{~mL} / \mathrm{min}-C r C L_{i}\right)\right) \cdot e^{\eta_{C L, i}},
\end{aligned}
$$

where $\mathrm{CrCL}_{i}$ is individual creatinine clearance expressed in $\mathrm{mL} / \mathrm{min}$ and $\theta$ is a 'shift parameters' describing the systematic dependence of drug clearance on individual creatinine clearance (other symbols: see the explanation to Eqs. 14.1-14.3). Covariate models should always be set up such that the mean population parameter represents the mean parameter value of typical individuals ('centering of covariates'); for example, in the model shown above, $\overline{C L}$ is the mean clearance of individuals with body weight $=70 \mathrm{~kg}$ and creatinine clearance $=120 \mathrm{~mL} / \mathrm{min}$. Expressions like: $C L_{i}=\left(\overline{C L} \cdot\left(\mathrm{BW}_{i} / 70\right)^{0.75}+\theta \cdot C r C L_{i}\right) \cdot e^{\eta_{C L, i}}$ should be avoided because they tend to generate correlations between parameter estimates ( $\widehat{C L}$ and $\hat{\theta}$ in the example) which will jeopardize the identification of potentially important covariates. The appropriateness of the functional form of the assumed covariate relationship should be checked by repeating the plot of individual ' $\eta$ 's' versus the observed covariate values. No trend should remain after including the covariate model.

Correlations between observed covariates, like a correlation between age and creatinine clearance, must be checked for by explorative plots. Only one of a pair of correlated covariates can be included in the model. Covariate relationships should be added to the model one at a time, the most likely ones first as suggested by pre-existing evidence. Covariate relationships which improve the fit significantly but were not expected from previous evidence should be documented as interesting new findings which may be confirmed in a subsequent trial.

\section{Goodness of Fit}

\section{Residuals}

The agreement between the final model and the data must be demonstrated by diagnostic plots; these plots are also helpful at all steps of the model-building process. A plot of the measured concentrations versus the 'mixed-effects predictions' must not show a systematic disagreement; the 'mixed-effects predictions', in this context, are those defined by the mean population parameters, individual body weight, other individual covariate values, and the Bayesian estimates of the individual random deviations from the mean population parameters (' $\eta$ 's', cf. Eq. 1). The residual variance model should be solved for $\varepsilon$; e.g. $\varepsilon=y_{i j} / \widehat{y_{i j}}-1$ 
for the multiplicative model (Eq. 7). This quantity should be computed for each data point and should be plotted versus time; it should be symmetrically distributed around zero without a time-dependence. If there is systematic disagreement between the data and the mixed-effects predictions, either with increasing concentration or with time, then the structural model is inadequate and must be revised, e.g. by adding a compartment, by adding a lag time parameter, by assuming zero-order rather than first-order absorption, or by introducing non-linearity.

\section{Individual Random Deviations From the Mean Population Parameters}

The individual random deviations from the mean population parameters $(\eta$ 's), as estimated by a Bayesian procedure, must be symmetrically distributed around zero. If they are not, then the mixed-effects predictions may still be adequate so that the structural model is acceptable (see preceding section). However, the estimated fixed-effects parameters (the mean population and the shift parameters) do not point to the central tendency of the data in this case and predictions of future outcomes based on these estimates would be misleading. Even the interindividual variance estimates are probably wrong (too large) in this situation resulting in false predictions of the variability of future outcomes, e.g. when the risk of exceeding a critical concentration, or the probability of staying below an effective concentration, are to be estimated. The interindividual variance model, including the modeled influence of covariates, must be revised then.

Plots of the distributions of the estimated individual $\eta$ 's can be of high explorative value by revealing distinct subgroups of patients with extraordinary kinetic behavior which were not expected from previous evidence. Sometimes these plots lead to the detection of false data that are still in the data set despite of the initial checks, or of errors resulting from special constellations in individual dosing or sampling histories that were not thought of when formatting the data for the model fitting program. Excluding patients or data points just because they are 'outliers' should be a last resort because such cases are likely to come up in future patient populations again. Such cases should be documented verbally.

\section{Simulation Check}

The residuals between the data and the fixed-effects predictions cannot be evaluated by inspection of plots alone. The reason is that they depend not only on residual variability but also on the distributions of the individual kinetic parameters which, in turn, depend one the distributions of random variables ( $\eta$ 's) as well as observed covariates. For each of the kinetic parameters, its interindividual distribution affects the residuals from the fixedeffects predictions in a different way, depending on where and how it occurs in the model equations. It is therefore practically impossible to evaluate the residuals from the fixed-effects predictions by plotting alone. This evaluation, however, is necessary in order to get an idea whether the estimated fixed-effects parameters correctly describe the data. A useful way to accomplish this evaluation is to simulate data based on the estimated population parameters: mean parameters, shift parameters, interindividual and residual variance parameters. The simulation must be done under the design of the original data set so that doses, administration times, sampling times, and covariate values are all the same for the observed and the simulated data. The entire set of the original observations may then be plotted versus time, along with summary statistics like the 5, 50, and 95\% quantiles; these statistics should coincide with the same statistics computed from the simulated data. The simulations may be repeated many times in order to judge to what extent discrepancies between the statistics of the simulated and the observed data are acceptable [19].

\section{References}

1 Ranson MR, Scarffe JH: Population and Bayesian pharmacokinetics in oncology. Clin Oncol 1994;6:254-260.

2 Rodman JH, Abromowitch M, Sinkule JA, Hayes FA, Rivera GK, Evans WE: Clinical pharmacodynamics of continuous infusion teniposide: Systemic exposure as a determinant of response in a phase I trial. J Clin Oncol 1987;5:1007-1014.

3 Milano G, Etienne MC, Renée N, Thyss A, Schneider M, Ramaioli A, Demard F: Rela- tionship between fluorouracil systemic exposure and tumor response and patient survival. J Clin Oncol 1994;12:1291-1295.

4 Evans WE, Relling MV, Rodman JH, Crom WR, Boyet JM, Pui CH: Conventional compared with individualized chemotherapy for childhood acute lymphoblastic leukemia. N Engl J Med 1998;338:499-505.

5 U.S. Department of Health and Human Services, Food and Drug Administration: Guidance for Industry · Population Pharma- cokinetics. http://www.fda.gov/cder/guidance/ 1852 fnl.pdf. 1999.

6 Vozeh S, Steimer JL, Rowland M, Morselli P, Mentré F, Balant LP, Aarons L: The use of population pharmacokinetics in drug development. Clin Pharmacokin 1996;30:81-93.

7 D'Argenio DZ: Optimal sampling times for pharmacokinetic experiments. J Pharmacokin Biopharm 1981;9:739-756.

8 D'Argenio DZ, Schumitzky A: ADAPT II, User's Guide. Biomedical Simulations Re- 
source, University of Southern California, Los Angles, 1997.

9 Karlsson MO, Sheiner LB: The importance of modeling interoccasion variability in population pharmacokinetic analyses. J Pharmacokin Biopharm 1995;21:735-750.

10 MathSoft Inc., Seattle/WA: S-Plus, version 6.0, release 1 for Linux 2.2.12 or higher, 2001.

11 Rowland M, Tozer TN: Clinical Pharmacokinetics: Concepts and Applications (3rd ed). Baltimore, Williams \& Wilkins, 1995.

12 Sheiner LB: Analysis of pharmacokinetic data using parametric models - 1: Regression models. J Pharmacokin Biopharm 1984; 12:98-117.

13 West GB, Brown JH, Enquist BJ: A general model for the origin of allometric scaling laws in biology. Science 1997;76:122-126.

14 Anderson BJ, McKee AD, Holford NHG: Size, myths and the clinical pharmacokinetics of analgesia in paediatric patients. Clin Pharmacokinet 1997;33:313-327.

15 Harrell, jr. FE: Regression Modeling Strategies. New York, Springer 2001.

16 Sheiner LB, Rosenberg B, Marathe VV: Estimation of population characteristics of pharmacokinetic parameters from routine clinical data. J Pharmacokin Biopharm 1977;5:445-479.

17 Sheiner LB: Analysis of pharmacokinetic data using parametric models. ii. Point estimates of an individual's parameters. J Pharmacokin Biopharm 1985;13:515-540.

18 Beal SL, Sheiner LB(eds): NONMEM version V.1.1, User's Guides. NONMEM Project Group, University of California San Francisco, San Francisco, CA, 1999.

19 Yano Y, Beal SL, Sheiner LB: Evaluating pharmacokinetic/pharmacodynamic models using the posterior predictive check. J Pharmacokin Pharmacodyn 2001;28:171-192. 